\title{
ON A MINIMUM PROBLEM IN THE THEORY OF ANALYTIC FUNCTIONS OF SEVERAL VARIABLES
}

\author{
BY
}

W. T. MARTIN

1. Introduction. In 1932 Wirtinger $\left(^{1}\right)$ posed and solved the following problem. Given a region $G$ in the complex $z$-plane and a (complex-valued) function $\phi(z, \bar{z}) \equiv \phi(x+i y, x-i y)$ continuous and with continuous first partial derivatives with respect to $x$ and $y$ in $G$, to find an analytic function $f(z)$ which gives the best approximation to $\phi$ in the mean-square sense, that is, such that

$$
\int_{G}|\phi-f|^{2} d \omega_{z}=\min
$$

where $d \omega_{z}$ is the element of area $d x d y$. (In the case in which $G$ extends to infinity he also assumed that $\phi \varepsilon L^{2}$ over $G$.) By use of the Green's function $G(z, \bar{z} ; \zeta, \bar{\zeta})$ for the region $G$, he proved the existence (and uniqueness) of such an $f$ and gave an explicit formula for $f$, namely,

$$
f(z)=\frac{1}{2 \pi} \int_{G} \phi(\zeta, \bar{\zeta}) \frac{\partial^{2} G}{\partial z \partial \bar{\zeta}} d \omega_{\zeta},
$$

when $\partial / \partial z \equiv \frac{1}{2}(\partial / \partial x-i \partial / \partial y), \partial / \partial \bar{z} \equiv \frac{1}{2}(\partial / \partial x+i \partial / \partial y)$, etc. In the case of the unit circle $C$ the formula yields the result

$$
f(z)=-\frac{1}{\pi} \int_{C} \frac{\phi(\zeta, \bar{\zeta})}{(1-z \bar{\zeta})^{2}} d \omega_{\zeta},
$$

a result which he also obtained directly by use of the Fourier series for $\phi$ and $f$.

Recently Wirtinger $\left({ }^{2}\right)$ posed the analogous question in the theory of functions of several complex variables. In the case in which the region under consideration is a hypersphere $H=E\left[\left|z_{1}\right|^{2}+\cdots+\left|z_{n}\right|^{2}<1\right]$ and $\phi\left(z_{1}, \cdots, z_{n}\right.$; $\bar{z}_{1}, \cdots, \bar{z}_{n}$ ) is merely integrable over $H$, he obtained a (unique) solution by use of multiple Fourier series, namely

$$
f\left(z_{1}, \cdots, z_{n}\right)=(-1)^{n} \frac{n !}{\pi^{n}} \int_{H} \frac{\phi\left(\zeta_{1}, \cdots, \zeta_{n} ; \bar{\zeta}_{1}, \cdots, \bar{\zeta}_{n}\right)}{\left[1-\left(z_{1} \bar{\zeta}_{1}+\cdots+z_{n} \bar{\zeta}_{n}\right)\right]^{n+1}} d \omega_{\zeta}
$$

Presented to the Society, October 28, 1939; received by the editors January 22, 1940. This paper was received by the editors of the Bulletin of the American Mathematical Society September 26, 1939, accepted by them, and later transferred to these Transactions.

(1) W. Wirtinger, Monatshefte für Mathematik und Physik, vol. 39 (1932), pp. 377-384.

(2) W. Wirtinger, Monatshefte für Mathematik und Physik, vol. 47 (1939), pp. 426-431. 
where $d \omega_{\zeta}$ is the $2 n$-dimensional volume element. He conjectured that the question probably has a solution for general regions and for very general functions $\phi$ but that the solution appeared to involve difficult investigations on the extensions of Green's functions. Now in various questions in the theory of functions of several complex variables Bergmann has been able to replace the theory of the Green's functions by the theory of complex orthogonal functions and the kernel of a region $\left({ }^{3}\right)$. In this note we show that by the use of this theory of the kernel of a region we can solve the problem posed by Wirtinger for a very general class of regions (which includes all bounded regions) and for $\phi$ belonging to $L^{2}$; indeed, we give the solution explicitly in terms of an integral involving $\phi$ and the kernel of the region (see equation (3.10)).

It is known that results of this general nature have important applications; for example in connection with the theory of entire functions of two variables Bergmann has solved the same problem with the function $f$ biharmonic (the real part of an analytic function of two variables) rather than analytic $\left(^{4}\right)$.

For the sake of completeness we shall give in $\$ 2$ a brief résumé of the results from the theory of orthogonal functions and the kernel of a region. Also in the concluding section we consider certain extensions of the problem. We shall speak only of two variables; the case of $n$ variables involves no essential changes.

2. The kernel of a region. To every region of a wide class of four-dimensional regions there corresponds a kernel function which is defined as follows $\left({ }^{5}\right)$. Let $\mathcal{B}$ be a region of this class and let $\left\{\Omega^{(v)}\left(z_{1}, z_{2}\right)\right\}$ be a complete orthonormal system of analytic functions belonging to $L^{2}$ over $\mathbb{B}$, so that

$$
\int_{\mathbb{B}} \Omega^{(\nu)}\left(z_{1}, z_{2}\right) \overline{\Omega^{(\mu)}\left(z_{1}, z_{2}\right)} d \omega_{z}=\delta_{\mu \nu}, \quad \mu, \nu=1,2, \cdots,
$$

where $\int \mathbb{B}=\lim _{m \rightarrow \infty} \int \mathcal{B}_{m}$ and $\left\{\mathcal{B}_{m}\right\}$ is a system of regions in $\mathbb{B}$ converging to $\mathbb{B}$. The series

$$
\sum_{\nu=1}^{\infty} \Omega^{(\nu)}\left(z_{1}, z_{2}\right) \overline{\Omega^{(v)}} \overline{\left.\zeta_{1}, \zeta_{2}\right)}
$$

(3) For the development of the theory of the kernel of a region see S. Bergmann, Mathematische Zeitschrift, vol. 29 (1929), pp. 640-677, Journal für die reine und angewandte Mathematik, vol. 169 (1933), pp. 1-42, especially pp. 1-5; vol. 172 (1934), pp. 89-128. We shall refer to these papers as $B_{1}$ and $B_{2}$ respectively.

(4) S. Bergmann, Mathematische Annalen, vol. 109 (1934), pp. 324-348, especially p. 333; Compositio Mathematica, vol. 3 (1934), pp. 137-173. We shall refer to these papers as $B_{3}$ and $\mathrm{B}_{4}$ respectively.

(5) The results which we state in this section are all given by Bergmann in the papers listed in footnotes 3 and 4 . We shall restrict ourselves to simply-connected bounded regions but the results are true for any region for which there exists a set of linearly independent functions belonging to $L^{2}$. 
converges absolutely and uniformly for $(z)$ and $(\zeta)$ in any regions interior to $B$ and accordingly defines a function of $z_{1}, z_{2}, \bar{\zeta}_{1}, \bar{\zeta}_{2}$ analytic for $(z)$ and $(\zeta)$ in $B$ (see $B_{2}$ ). The sum function is called the kernel of the region $B$ and is denoted by $K_{\mathrm{B}}\left(z_{1}, z_{2}, \bar{\zeta}_{1}, \bar{\zeta}_{2}\right)$. It is known that the function depends only upon the region $B$ and not upon the particular set of orthonormal functions used in defining it (see $\mathrm{B}_{2}$ ). Concerning series in terms of the $\Omega^{(v)}$ it has been shown that the series

$$
\left|\sum_{\nu=1}^{\infty} a_{\nu} \Omega^{(\nu)}\left(z_{1}, z_{2}\right)\right|^{2}
$$

can be integrated term-by-term over $\mathbb{B}$ whenever $\sum\left|a_{\nu}\right|^{2}<\infty$ (see $B_{3}$, p. 331).

3 . Solution of the problem. Let $\phi\left(z_{1}, z_{2} ; \bar{z}_{1}, \bar{z}_{2}\right)$ be a complex-valued function of the four real variables $x_{1}, x_{2}, y_{1}, y_{2}$, defined and of integrable square over a bounded region $B$

$$
\int_{\mathcal{B}}|\phi|^{2} d \omega_{z}<\infty
$$

We seek a function $f\left(z_{1}, z_{2}\right)$ analytic and of integrable square over $\mathbb{B}$ and such that

$$
\int_{\mathcal{B}}|\phi-f|^{2} d \omega_{z}=\min
$$

For the solution let $\left\{\Omega^{(\nu)}\left(z_{1}, z_{2}\right)\right\}$ be a complete orthonormal set of analytic functions belonging to $L^{2}$ over $B$ and let us seek to determine coefficients $\left\{a_{\nu}\right\}$ subject to the condition

$$
\sum_{1}^{\infty}\left|a_{\nu}\right|^{2}<\infty
$$

in such a manner that the function

$$
f\left(z_{1}, z_{2}\right)=\sum_{1}^{\infty} a_{\nu} \Omega^{(\nu)}\left(z_{1}, z_{2}\right)
$$

furnishes a minimum to (3.2). If we substitute (3.4) into (3.2) we find

$$
\begin{aligned}
\int_{\mathcal{B}}|\phi-f|^{2} d \omega= & \int_{\mathcal{B}}|\phi|^{2} d \omega-\int_{\mathcal{B}}\left(\sum_{1}^{\infty} a_{\nu} \Omega^{(v)}\right) \bar{\phi} d \omega \\
& -\int_{\mathcal{B}}\left(\sum_{1}^{\infty} \bar{a}_{\nu} \overline{\Omega^{(v)}}\right) \phi d \omega+\int_{\mathcal{B}}\left|\sum_{1}^{\infty} a_{\nu} \Omega^{(v)}\right|^{2} d \omega .
\end{aligned}
$$

Using (3.3) and the results stated in $\$ 2$ we see that we may integrate termwise, thus 


$$
\int_{\mathcal{B}}|\phi-f|^{2} d \omega=\int_{\mathcal{B}}|\phi|^{2} d \omega-\sum_{1}^{\infty}\left(a_{\nu} \bar{b}_{\nu}+\bar{a}_{\nu} b_{\nu}-a_{\nu} \bar{a}_{\nu}\right),
$$

where we have written

$$
b_{\nu}=\int_{\mathscr{B}} \phi \overline{\Omega^{(\nu)}} d \omega
$$

By Bessel's inequality

$$
\sum_{\nu=1}^{\infty}\left|b_{\nu}\right|^{2}<\infty
$$

Treating $a_{\nu}, \bar{a}_{\nu}$ as independent complex variables and differentiating with respect to $\bar{a}_{\nu}\left(\right.$ or $\left.a_{v}\right)$ we see that Euler's conditions for (3.6) to be a minimum are

$$
a_{\nu}=b_{\nu}, \quad \quad \nu=1,2, \cdots .
$$

Clearly this choice of the $a$ 's furnishes an actual minimum (we shall also give a direct proof of this fact in equation (3.12) below). Thus the minimizing function $f$ has the form

$$
\begin{aligned}
f\left(z_{1}, z_{2}\right) & =\sum_{1}^{\infty} \Omega^{(v)}\left(z_{1}, z_{2}\right) \int_{\mathcal{B}} \phi\left(\zeta_{1}, \zeta_{2} ; \bar{\zeta}_{1}, \bar{\zeta}_{2}\right) \overline{\Omega^{(v)}\left(\zeta_{1}, \zeta_{2}\right)} d \omega_{\zeta} \\
& =\int_{\mathcal{B}} \phi\left(\zeta_{1}, \zeta_{2} ; \bar{\zeta}_{1}, \bar{\zeta}_{2}\right) \sum_{1}^{\infty} \Omega^{(v)}\left(z_{1}, z_{2}\right) \overline{\Omega^{(v)}\left(\zeta_{1}, \zeta_{2}\right)} d \omega_{\zeta} \\
& =\int_{\mathcal{B}} \phi\left(\zeta_{1}, \zeta_{2}, \bar{\zeta}_{1}, \bar{\zeta}_{2}\right) K_{\mathcal{B}}\left(z_{1}, z_{2}, \bar{\zeta}_{1}, \bar{\zeta}_{2}\right) d \omega_{\zeta}
\end{aligned}
$$

where we have again used the fact that we may interchange the order of integration and summation.

Thus we have answered Wirtinger's question.

THEOREM. Let $\mathbb{B}$ be any (four-dimensional) region for which there exists an infinite system of linearly independent analytic functions of $L^{2}$. (In particular, let $\mathcal{B}$ be any simply connected bounded region.) Let $\phi\left(z_{1}, z_{2}, \bar{z}_{1}, \bar{z}_{2}\right)$ be of integrable square over $B$. Then the function $f$ defined by

$$
f\left(z_{1}, z_{2}\right)=\int_{\mathcal{B}} \phi\left(\zeta_{1}, \zeta_{2} ; \bar{\zeta}_{1}, \bar{\zeta}_{2}\right) K_{\mathcal{B}}\left(z_{1}, z_{2}, \bar{\zeta}_{1}, \bar{\zeta}_{2}\right) d \omega_{\zeta}
$$

where $K B$ is the kernel of the region $B$ defined as in (2.2), is analytic and of integrable square over $\mathbb{B}$ and furnishes the unique minimum to the integral (3.2) over the class of analytic functions of integrable square.

Very many different properties of the kernel function are known which 
yield various properties of the minimizing function $f$; for example if $g\left(z_{1}, z_{2}\right)$ is any analytic function of $L^{2}$ over $\mathbb{B}$ then $\left({ }^{6}\right)$

$$
\int_{\mathcal{B}}(\phi-f) \bar{g} d \omega_{z}=0
$$

This result is the analogue for the region $B$ of a result obtained by Wirtinger for the hypersphere (loc. cit., footnote 2, equation (8)). It also obviously furnishes a direct proof of the fact that the function $f$ defined in (3.10) yields a minimum for (3.2), since in view of (3.11), if $g \neq 0$,

$$
\begin{aligned}
\int|f+g-\phi|^{2} & =\int|f-\phi|^{2}+\int(f-\phi) \bar{g}+\int(\bar{f}-\bar{\phi}) g+\int|g|^{2} \\
& =\int|f-\phi|^{2}+\int|g|^{2}>\int|f-\phi|^{2} .
\end{aligned}
$$

4. Special regions. For many special regions the kernel function has been given explicitly, for instance in the case of a Reinhardt region in four-dimensional space

$$
R=E\left[\left|z_{2}\right|^{2}<G\left(\left|z_{1}\right|^{2}\right), \quad 0 \leqq\left|z_{1}\right|<1\right],
$$

where $G$ is once differentiable in $(0,1)$, the kernel has the form (see $\mathrm{B}_{1}$ )

$$
K_{R}\left(z_{1}, z_{2}, \bar{\zeta}_{1}, \bar{\zeta}_{2}\right)=\sum_{m=0}^{\infty} \sum_{p=0}^{\infty} \frac{z_{1}^{m} \bar{\zeta}_{1}^{m} z_{2}^{p} \bar{\zeta}_{2}^{p}}{\left[\pi^{2} /(p+1)\right] \int_{0}^{1} \rho^{m}[G(\rho)]^{p+1} d \rho}
$$

If we have a region $R^{*}$ which can be mapped into $R$ by means of a transformation $z_{\kappa}=z_{\kappa}\left(w_{1}, w_{2}\right), \kappa=1,2$, where the $z_{\kappa}$ are analytic in $R^{*}$, then the kernel function for $R^{*}$ is equal to the kernel for $R$ multiplied by the two jacobians of the transformation (see $B_{1}$, p. 5):

$$
\begin{aligned}
K_{R^{*}}\left(w_{1}, w_{2}, \bar{\xi}_{1}, \bar{\xi}_{2}\right)= & {\left[K_{R}\left(z_{1}\left(w_{1}, w_{2}\right), z_{2}\left(w_{1}, w_{2}\right), \overline{z_{1}\left(\xi_{1}, \xi_{2}\right)}, \overline{z_{2}\left(\xi_{1}, \xi_{2}\right)}\right)\right.} \\
& \left.\cdot \frac{D\left(z_{1}, z_{2}\right)}{D\left(w_{1}, w_{2}\right)} \frac{\overline{D\left(z_{1}, z_{2}\right)}}{D\left(\xi_{1}, \xi_{2}\right)}\right]
\end{aligned}
$$

In different cases the series in (4.2) can be summed, for example in the

${ }^{(6)}$ We may see this fact directly in view of the form of $f$ and the orthogonality of the $\Omega$ 's, or we may note that the corresponding result for the case of biharmonic functions has been proved by Bergmann (see $B_{3}$, p. 333). In order to see it directly let us write $c_{\nu}=\int g \overline{\Omega^{(\nu)}}$. Then $g\left(z_{1}, z_{2}\right)=\sum_{1}^{\infty} c_{\nu} \Omega^{(\nu)}\left(z_{1}, z_{2}\right)$. Also by (3.4), (3.7) and (3.9) $f\left(z_{1}, z_{2}\right)=\sum_{1}^{\infty} b_{\nu} \Omega^{(\nu)}\left(z_{1}, z_{2}\right)$ where $b_{\nu}$ $=\int \phi \overline{\Omega^{(v)}}$. Thus

$$
\begin{aligned}
\int(\phi-f) \bar{g} & =\int \phi \sum \bar{c}_{\nu} \overline{\Omega^{(\nu)}}-\int\left[\sum b_{n} \Omega^{(n)}\right]\left[\sum \overline{c_{\nu}} \overline{\Omega^{(\nu)}}\right] \\
& =\sum \bar{c}_{\nu} \int \phi \overline{\Omega^{(v)}}-\sum \bar{c}_{\nu} b_{\nu}=0 .
\end{aligned}
$$


case of a region of the form

$$
a\left|z_{1}\right|^{2 / p}+\left|z_{2}\right|^{2}<1, \quad \quad p \text { integral, } p>0,0<a \leqq 1,
$$

the kernel is (see $B_{1}$ )

$$
K\left(z_{1}, z_{2} ; \bar{\zeta}_{1}, \bar{\zeta}_{2}\right)=a^{p}\left(1-z_{2} \bar{\zeta}_{2}\right)^{p-2} \frac{(p+1)\left(1-z_{2} \bar{\zeta}_{2}\right)^{p}+(p-1) a^{p} z_{1} \bar{\zeta}_{1}}{\pi^{2}\left[\left(1-z_{2} \bar{\zeta}_{2}\right)^{p}-a^{p} z_{1} \bar{\zeta}_{1}\right]^{3}}
$$

which yields for the hypersphere $H=E\left[\left|z_{1}\right|^{2}+\left|z_{2}\right|^{2}<1\right]$ the result

$$
K_{H}\left(z_{1}, z_{2} ; \bar{\zeta}_{1}, \bar{\zeta}_{2}\right)=\frac{2}{\pi^{2}\left[1-z_{1} \bar{\zeta}_{1}-z_{2} \bar{\zeta}_{2}\right]^{3}} .
$$

If we put this into (3.10), then we see that for the hypersphere $H$ our result is identical with the formula (1.3) obtained by Wirtinger (for $n=2$ ).

It is perhaps worth while merely to mention that in the case of a bicylinder $\left|z_{\kappa}\right|<r_{\kappa}, \kappa=1,2$, the kernel has the form (see $B_{1}$ )

$$
K\left(z_{1}, z_{2}, \bar{\zeta}_{1}, \bar{\zeta}_{2}\right)=\frac{r_{1}^{2} r_{2}^{2}}{\pi^{2}\left(r_{1}^{2}-z_{1} \bar{\zeta}_{1}\right)^{2}\left(r_{1}^{2}-z_{2} \bar{\zeta}_{2}\right)^{2}} .
$$

It is also interesting that in the case of simply connected regions in the complex $z$-plane the kernel is simply the expression $\partial^{2} G(z, \bar{\zeta}) / \partial z \partial \bar{\zeta}$ where $G$ is the Green's function for the region $\left({ }^{7}\right)$. This of course is in agreement with the result (1.2) of Wirtingers' mentioned in the introduction.

5. Extensions. A very important variation of the problem in the theory of functions of one complex variable is the case in which the integration is over the boundary curve. In the case of two complex variables, when the region under consideration has a distinguished boundary surface, the analogous problem may be solved and since there is a general theory of orthogonal functions and kernel functions related to the distinguished boundary surface $\left.{ }^{8}\right)$, the same formula for $f$ as in (3.10) is obtained, with of course the kernel $K$ defined analogously.

Moreover we may ask not only that $f$ be analytic and of $L^{2}$ over $\mathcal{B}$ and minimize the integral (3.2) but also that $f$ be subjected to certain additional conditions, for example that

$$
f\left(t_{1}^{(s)}, t_{2}^{(s)}\right)=X_{s}, \quad s=1, \cdots, p,
$$

( 7 ) The kernel for doubly connected regions in the complex z-plane has been calculated by K. Zarankiewicz, Zeitschrift für angewandte Mathematik und Mechanik, vol. 14 (1934), pp. 97-104 and by P. Kufareff, Bulletin de l'Institut Mathématique et Méchanique, Tomsk, vol. 1 (1937), pp. 228-235.

(8) See Bergmann, Bulletin de l'Institut Mathématique et Méchanique, Tomsk, vol. 3 (1935-1937), pp. 242-257. 
where $\left\{t_{1}^{(s)}, t_{2}^{(s)}\right\} \in \mathcal{B}$. We shall merely indicate the proof in the case $p=1$. Our problem is then to find an $f$ analytic and of $L^{2}$ over $B$, which minimizes the integral (3.2) and which takes on a given value $X$ at a fixed point $\left(t_{1}, t_{2}\right)$ in $\mathcal{B}$,

$$
f\left(t_{1}, t_{2}\right)=X \text {. }
$$

The analogue of $(3.6)$ is

$$
\begin{aligned}
\int|\phi-f|^{2}-\lambda[f(t)-X]-\mu[\overline{f(t)}-\bar{X}] \\
=\int|\phi|^{2}-\sum_{1}^{\infty}\left(a_{\nu} b_{\nu}+a_{\nu} \bar{b}_{\nu}-a_{\nu} \bar{a}_{\nu}\right)-\lambda\left[\sum_{1}^{\infty} a_{\nu} \Omega^{(\nu)}(t)-X\right] \\
\quad-\mu\left[\sum \bar{a}_{\nu} \bar{\Omega}^{(\nu)}(t)-\bar{X}\right]
\end{aligned}
$$

where $\lambda, \mu$ are the Lagrangian multipliers. Euler's conditions are

$$
a_{\nu}=b_{\nu}+\mu \overline{\Omega^{(\nu)}(\bar{t})}, \quad \bar{a}_{\nu}=\bar{b}_{\nu}+\lambda \Omega^{(\nu)}(t), \quad \nu=1,2, \cdots .
$$

Thus $\mu=\bar{\lambda}$ and the condition (5.2) yields

$$
\mu=\frac{X-\sum_{1}^{\infty} b_{\nu} \Omega^{(v)}(t)}{K_{\mathcal{B}}(t, \bar{t})} .
$$

Thus the minimizing function $f$ has the form

$$
\begin{aligned}
f\left(z_{1}, z_{2}\right)= & \int_{\mathcal{B}} \phi\left(\zeta_{1}, \zeta_{2} ; \bar{\zeta}_{1}, \bar{\zeta}_{2}\right) K_{\mathcal{B}}\left(z_{1}, z_{2} ; \bar{\zeta}_{1}, \bar{\zeta}_{2}\right) d \omega_{\zeta} \\
& +\frac{X-\int_{\mathcal{B}} \phi\left(\zeta_{1}, \zeta_{2}, \bar{\zeta}_{1}, \bar{\zeta}_{2}\right) K_{\mathcal{B}}\left(t_{1}, t_{2} ; \bar{\zeta}_{1}, \bar{\zeta}_{2}\right) d \omega_{\zeta}}{K_{\mathcal{B}}\left(t_{1}, t_{2} ; \bar{t}_{1}, \bar{t}_{2}\right)} K_{\mathcal{B}}\left(z_{1}, z_{2} ; \bar{t}_{1}, \bar{t}_{2}\right)
\end{aligned}
$$

Massachusetts Institute of Technology,

Cambridge, Mass. 